\title{
Cryo-EM Structure of the Novel Viral Chaperonin, Encoded by Gene 228 of Bacteriophage AR9 Bacillus subtilis
}

\author{
Olga Sokolova ${ }^{1}$, Evgeny Pichkur $^{2}$ and Lidia Kurochkina ${ }^{3}$ \\ ${ }^{1}$ Lomonosov Moscow State University, Faculty of Biology, Moscow, Moskva, Russia, ${ }^{2}$ Kurchatov \\ Institute, Moscow, Moskva, Russia, ${ }^{3}$ Belozersky Research Institute of Physico-Chemical Biology, \\ Lomonosov Moscow State University, Moscow, Moscow, Moskva, Russia
}

Chaperonins are some of the most studied families of chaperones. They are multimeric complexes that ensure the proper folding of newly synthesized polypeptide chains and prevent the aggregation of denatured stressed proteins in an ATP-dependent manner. Chaperonins are divided into two groups: group I, found in eubacteria and in endosymbiotic organelles, and group II, found in archaea and the cytosol of eukaryotes [1]. Recently, we demonstrated that some viruses of bacteria (bacteriophages) also encode GroEL-like proteins, which possess chaperonin properties [2-4]. With the addition of new annotated bacteriophage genomes to the database [4,5], the number of predicted GroEL-like proteins has increased. An analysis of their structural and functional characteristics may provide important insights into the diversity of phage chaperonins.

Here, we have expressed and structurally characterized the new putative GroEL-like protein encoded by gene 228, predicted in the genome of bacteriophage AR9 Bacillus subtilis. Recombinant gp228 was precipitated from supernatant by increasing the concentration of ammonium sulfate to $50 \%$. The protein precipitate was pelleted by centrifugation, dissolved in $50 \mathrm{mM}$ Tris- $\mathrm{HCl}(\mathrm{pH} 7.5), 100 \mathrm{mM} \mathrm{NaCl}$ and fractionated on a Q-sepharose (Bio-Rad, USA) column in a linear gradient from 100 to $500 \mathrm{mM} \mathrm{NaCl}$ in the same buffer. The concentrated fraction, containing the pure protein was loaded on the SDS-PAGE (Fig. 1A). For cryo-EM, 3ul of the purified sample were applied to freshly glow-discharged grids (Quantifoil R1.2/1.3) and vitrified in a Vitrobot Mark IV at $4.5^{\circ} \mathrm{C}$. Cryo-EM data collection was performed using a Thermo-Fisher Krios equipped with a direct electron detector Falcon II. 3182 movie stacks were collected in total, followed by motion correction, CTF estimation, and particle picking in Warp [6]. CryoEM data processing was performed utilizing the CryoSPARC [7]. Over one million of the particles were used for 2D and 3D classification, resulting in approximately 331000 particles used for the final reconstruction to solve the structure at an average resolution of $3.7 \AA$ (Fig. 1B).

The structure of gp228 chaperonin resembles a single ring conformation in the APO state, which is unusual for most known chaperonins. A similar structure has recently been determined for another chaperonin of the bacteriophage OBP Pseudomonas fluorescens [8]. While the OBP chaperonin reveals a unique subunit arrangement, with three subunits pairs and one unpaired subunit, gp228 chaperonin demonstrates less flexibility (Fig. 1C). High-resolution features at the equatorial domains of gp228 were clearly visible, while apical domains were not clearly resolved, implying high flexibility of this region. We proposed that the high flexibility of the subunits is required to protect substrate proteins from aggregation since it is likely that gp228 chaperonin, similar to OBP chaperonin [8], is functional in the form of a single ring [10]. 

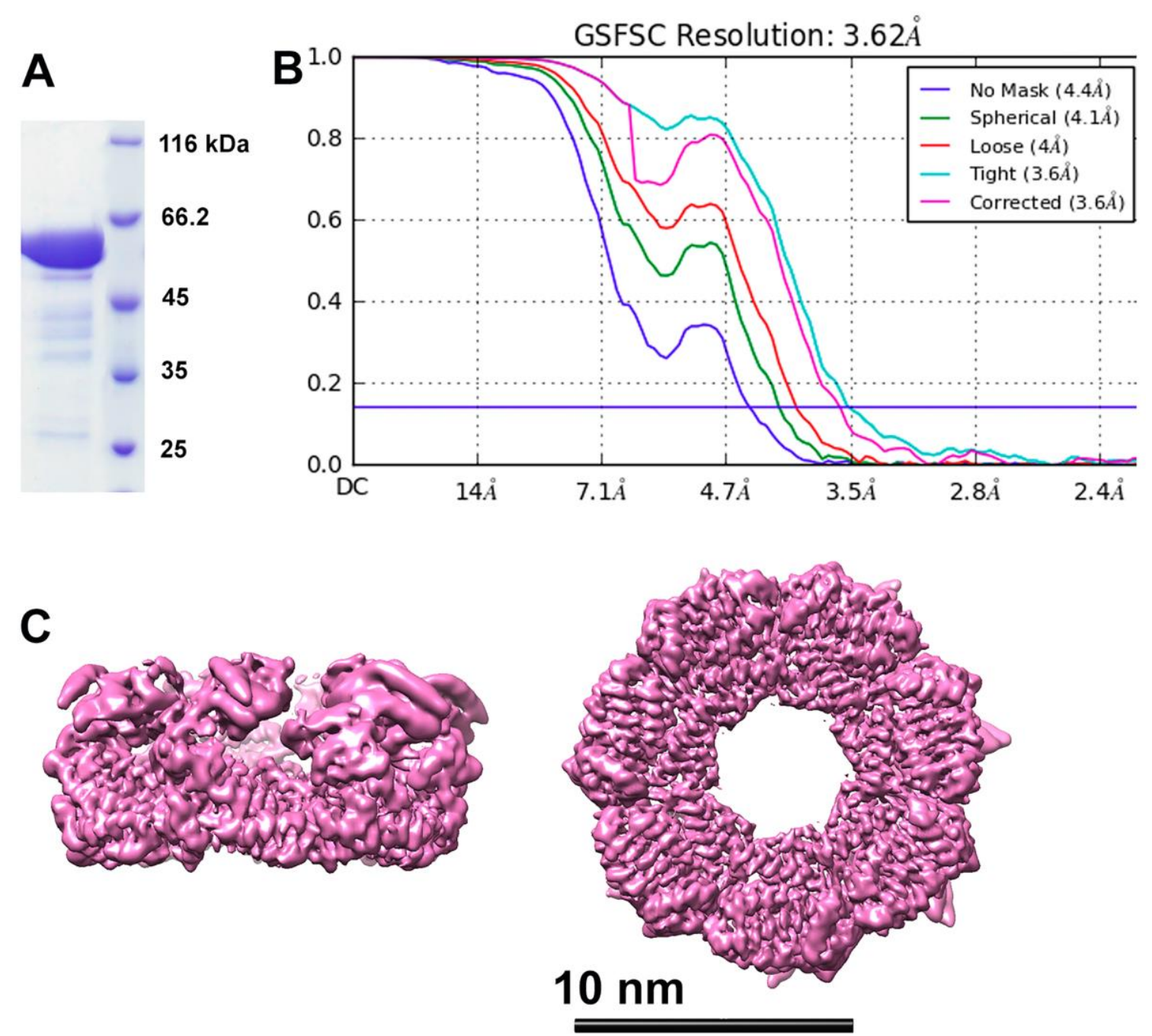

Figure 1. Cryo-EM structure of gp228 chaperonin. (A) SDS-10\% PAGE of recombinant gp246, after precipitation with $30 \%$ ammonium sulfate; (B) FSC curves of the reconstruction between two independently refined half maps before (red) and after (blue) masking, indicated with resolutions corresponding to $\mathrm{FSC}=0.143$; (C) 3D density map of the nucleotide-free state of gp228 chaperonin. Left - side view, right - top view (bar size - $10 \mathrm{~nm}$ )

\section{References}

[1] Ranson, N.A. et al, Biochem J. 333 (1998) 233-242.

[2] Hertveldt, K. J. et al, Mol. Biol. 354 (2005) 536-545.

[3] Kurochkina, L.P. et al, J. Virol. 86 (2012) 10103-10111.

[4] Semenyuk, P.I. et al,Biochemical Journal. 473 (2016) 2383-2393.

[5] Cornelissen, A. et al, J. Virol. 86 (2012) 1844-1852.

[6] Zheng, S. Q. et al. Nat. Methods 4-5 (2017).

[7] Mindell, J. A. \& Grigorieff, N. J. Struct. Biol. 142, 334-347 (2003).

[8] Punjanji, A. et al., Nat. Methods 14, 290-296 (2017)

[9] Stanishneva-Konovalova, T.B., et al. Journal of Structural Biology (2019): 107439.

[10] This work was supported by the Russian Fund for Basic Research (\#19-04-00605 to O.S.S. - cryoEM and data processing; \#18-04-01281 to L.P.K. - protein expression and purification). 